Please do not remove this page

RMIT

UNIVERSITY

\title{
Optical cryocooling of diamond
}

Kern, M.; Jeske, Jan; Lau, Desmond; Greentree, Andrew; Jelezko, Fedor; Twamley, J.

https://researchrepository.rmit.edu.au/esploro/outputs/9921860522801341/filesAndLinks?institution=61RMIT_INST\&index=null

Kern, M., Jeske, J., Lau, D., Greentree, A., Jelezko, F., \& Twamley, J. (2017). Optical cryocooling of diamond. Physical Review B, 95, 1-8. https://doi.org/10.1103/PhysRevB.95.235306

Document Version: Published Version

Published Version: https://doi.org/10.1103/PhysRevB.95.235306

Repository homepage: https://researchrepository.rmit.edu.au

(C)2017 American Physical Society

Downloaded On 2023/04/26 20:26:48 +1000

Please do not remove this page 
Thank you for downloading this document from the RMIT Research Repository.

The RMIT Research Repository is an open access database showcasing the research outputs of RMIT University researchers.

RMIT Research Repository: http://researchbank.rmit.edu.au/

\author{
Citation: \\ Kern, M, Jeske, J, Lau, D, Greentree, A, Jelezko, F and Twamley, J 2017, 'Optical \\ cryocooling of diamond', Physical Review B, vol. 95, 235306, pp. 1-8.
}

See this record in the RMIT Research Repository at:

https://researchbank.rmit.edu.au/view/rmit:43736

Version: Published Version

Copyright Statement:

(C) 2017 American Physical Society

Link to Published Version:

https://dx.doi.org/10.1103/PhysRevB.95.235306 


\title{
Optical cryocooling of diamond
}

\author{
M. Kern, ${ }^{1,2}$ J. Jeske, ${ }^{3}$ D. W. M. Lau, ${ }^{3,4}$ A. D. Greentree,,${ }^{3,4}$ F. Jelezko, ${ }^{5,6}$ and J. Twamley ${ }^{7}$ \\ ${ }^{1}$ Ulm University, Ulm D-89073, Germany \\ ${ }^{2}$ Department of Physics and Astronomy, Macquarie University, Sydney, New South Wales 2109, Australia \\ ${ }^{3}$ Chemical and Quantum Physics, School of Applied Sciences, RMIT University, Melbourne, Victoria 3001, Australia \\ ${ }^{4}$ ARC Centre of Excellence for Nanoscale BioPhotonics, RMIT University, Melbourne, Victoria 3001, Australia \\ ${ }^{5}$ Institute of Quantum Optics, Ulm University, 89081 Ulm, Germany \\ ${ }^{6}$ Center for Integrated Quantum Science and Technology (IQST), Ulm University, 89081 Ulm, Germany \\ ${ }^{7}$ Centre for Engineered Quantum Systems, Department of Physics and Astronomy, Macquarie University, \\ Sydney, New South Wales 2109, Australia \\ (Received 23 January 2016; published 21 June 2017)
}

\begin{abstract}
The cooling of solids by optical means only using anti-Stokes emission has a long history of research and achievements. Such cooling methods have many advantages ranging from no moving parts or fluids through to operation in vacuum and may have applications to cryosurgery. However, achieving large optical cryocooling powers has been difficult to manage except in certain rare-earth crystals but these are mostly toxic and not biocompatible. Through study of the emission and absorption cross sections we find that diamond, containing either nitrogen vacancy (NV) or silicon vacancy defects, shows potential for optical cryocooling and, in particular, $\mathrm{NV}$ doping shows promise for optical refrigeration. We study the optical cooling of doped diamond microcrystals ranging $10-250 \mu \mathrm{m}$ in diameter trapped either in vacuum or in water. For the vacuum case we find NV-doped microdiamond optical cooling below room temperature could exceed $|\Delta T|>10 \mathrm{~K}$ for irradiation powers of $P_{\text {in }}<100 \mathrm{~mW}$. We predict that such temperature changes should be easily observed via large alterations in the diffusion constant for optically cryocooled microdiamonds trapped in water in an optical tweezer or via spectroscopic signatures such as the zero-phonon line width or Raman line.
\end{abstract}

DOI: 10.1103/PhysRevB.95.235306

Optical refrigeration, or optical cryocooling, uses antiStokes emission in solids to deplete the phonon population within the solid, cooling it. First suggested by Pringsheim [1], and initially demonstrated by Epstein et al. in 1995 [2], the phenomenon is observed when low-entropy laser light is primarily absorbed at wavelengths slightly longer than the mean fluorescence wavelength $\left(\lambda_{f}\right)$ of the material. The light is reemitted with a broadband fluorescence possessing a mean energy which is higher than the incident pump laser. The increase in energy is due to absorption of lattice phonons (vibrations), reducing the net temperature of the solid. Optical refrigeration has experimentally achieved temperatures of $T \sim 155 \mathrm{~K}$ (from room temperature) using ytterbium-doped fluoride crystal $\left(\mathrm{YLiF}_{4}: \mathrm{Yb}^{3+}\right)$ [3], $T \sim 250 \mathrm{~K}$ (from $290 \mathrm{~K}$ ) for the semiconductor CdS [4], and $T \sim 114 \mathrm{~K}$ more recently using a multipass setup with $\mathrm{Yb}$-doped crystals [5]. These temperatures outperform Peltier or thermoelectric coolers and reach into the defined cryogenic regime $(<123 \mathrm{~K})$. Importantly they operate with no mechanical vibrations, magnetic or electric fields, or moving mechanical, liquid, or gas components and are ideal refrigeration solutions in many difficult or sensitive situations, e.g., optomechanical, space, and sensing experiments [6-9]. In addition, spin properties of diamond defects have recently attracted much attention owing to their long spin coherence times $T_{2}$. Since $T_{2}$ increase at low temperatures, a new way of cooling diamond is important for applications where long spin-relaxation times are needed. Microscopic diamond crystals are highly biocompatible and can be functionalized to attach to specific biologically important ligands [10]. Cryosurgery or cryotherapy is a method to destroy diseased or harmful tissues within the body and involves cyclic freezing and thawing of the cells involved [11-13]. Developing optical methods of cooling nanoscopic diamonds located in diseased cells to cause cell death may provide a novel route towards targeted cryosurgery.

In the following we consider a primarily theoretical investigation of the capability of optical refrigeration using diamond and, in particular, using nitrogen-vacancy (NV) or silicon-vacancy (SiV) defects in diamond. By estimating, via experiment and theory, the emission and absorption cross sections for these two defects over a range of wavelengths, we are able to estimate the optical cryocooling power based on a suitable two-level model. We predict that cooling can be achieved using both defects; however, the NV defect promises very significant cooling due to its high quantum efficiency. We consider the optical cryocooling of NV- or SiV-doped microdiamonds (MDs) and find substantial temperature changes depending on the irradiation laser power, wavelength, and quantum efficiency of the defect. We show that it is possible to measure the temperature change $\Delta T$ in a MD (diameter $<200 \mu \mathrm{m}$ ), particularly if it is held in three dimensions in a trap (e.g., an optical or electrodynamic trap). There are various means of inferring the MD temperature, either via temperature-dependent spectroscopic features, e.g., the zerophonon-line (ZPL) width, or the diamond Raman linewidth. Another signature when the microdiamond is trapped in liquid is the temperature dependence of the particle's diffusion rate.

\section{MODEL}

Models of optical refrigeration include a four-level [14] and two-level model [15]. We note that the efficiency of optical cryocooling is degraded when there are any nonradiative routes for the optically excited state to decay through. The latter 
will mostly heat the material. In the following we find that indeed the achieved temperature change is very sensitive to the overall quantum efficiency of the emitter. We focus on the two-level model of [15]. In this model one considers a coherent laser beam of circular cross section (radius $r_{s}$ and area $\alpha_{\text {eff }}$ ), with power $P$ and wavelength $\lambda$, which passes through a cylinder (with diameter $d$ and length $L$ ) of cryocooling material containing an ensemble of two-level systems with a defect number density $N=n_{\downarrow}+n_{\uparrow}$, consisting of defects in the optical ground (excited) state $n_{\downarrow}\left(n_{\uparrow}\right)$, with optical decay rate $\gamma_{\mathrm{rad}}$. Assuming the two-level systems exhibit wavelength-dependent absorption and spontaneous emission cross sections $\sigma_{\text {abs }}(\lambda)$ and $\sigma_{\text {se }}(\lambda)$, one has a saturation intensity $I_{S}(\lambda)=h c \gamma_{\mathrm{rad}} /\left[\lambda \sigma_{\mathrm{abs}}(\lambda)\right]$, and mean fluorescence emission wavelength $\lambda_{F}=\int \lambda \sigma_{\mathrm{se}}(\lambda) d \lambda / \int \sigma_{\mathrm{se}}(\lambda) d \lambda$. Considering the steady-state occupation and assuming the material is optically thin over the range of wavelengths of interest, one obtains the cooling power as [15]

$$
P_{\text {cool }}=N L \alpha_{\mathrm{eff}} I_{S} \frac{\sigma_{\mathrm{abs}}\left(\lambda / \lambda_{F^{*}}-1\right)}{1+\sigma_{\mathrm{se}} / \sigma_{\mathrm{abs}}+\alpha_{\mathrm{eff}} I_{S} / P},
$$

where $\lambda_{F^{*}}=\left[1 / \lambda_{F}-\kappa /\left(h c \gamma_{\mathrm{rad}}\right)\right]^{-1}$ incorporates any heating $\kappa$, generated by nonradiative decay or other processes in the diamond, e.g., Raman scattering. In the case of the NV defect the branching of the decay of the optical excited state through nonradiative paths can be reduced greatly by exciting from the $\left|m_{s}=0\right\rangle$ ground state. Since one can optically pump the $\mathrm{NV}$ into this state with high efficiency before initializing the cooling we first assume $\kappa \sim 0$. We also expect that the long-wavelength irradiation required for cryocooling will continue to pump the NVs into this ground state. We now consider a roughly spherical diamond particle which is held in a three-dimensional (3D) trap. To estimate the change in temperature (the cooling region of the laser and diamond is cylindrical), one must determine the thermal load experienced by the particle, and this depends on the physical surroundings of the particle. In [15], the primary thermal load arises from blackbody absorption of ambient room-temperature radiation by the cold particle. Assuming the change in temperature of the material $\Delta T=T_{\text {mat }}-T_{\mathrm{amb}}$ is small as compared with the ambient temperature, then one has

$$
P_{\text {load }} \approx-4 A \epsilon_{\mathrm{eff}} \sigma_{B} T_{\mathrm{amb}}^{3} \Delta T,
$$

where $\sigma_{B}$ is the Stefan-Boltzmann constant, $\epsilon_{\text {eff }}$ is the effective emissivity of the cylinder, i.e., the fraction of energy emitted or absorbed relative to that emitted by a thermal blackbody, and $A$ is the surface area of the spherical diamond particle of diameter $D$, which is illuminated by the laser which passes through the center of the sphere [yielding $L=D$ in Eq. (1)]. We underestimate $\Delta T$ by assuming $\epsilon_{\text {eff }}=1$. Combining Eqs. (1) and (2) we obtain

$$
\Delta T=\frac{N \alpha_{\mathrm{eff}} I_{S}}{4 \pi D \epsilon_{\mathrm{eff}} \sigma_{B} T_{\mathrm{amb}}^{3}} \frac{\sigma_{\mathrm{abs}}\left(1-\lambda / \lambda_{F}\right)}{1+\sigma_{\mathrm{se}} / \sigma_{\mathrm{abs}}+\alpha_{\mathrm{eff}} I_{S} / P} .
$$

In [15] researchers studied the optical cryocooling of Zirconium Barium Lanthanum Aluminum Sodium Lead Fluoride (ZBLANP) glass doped with $\mathrm{Yb}^{3+}$ ions in a cylindrical optical fiber with diameter $250 \mu \mathrm{m}$ and demonstrated cooling of the fiber to $21 \mathrm{~K}$ below room temperature. Crucial towards achieving this are the detailed spontaneous emission and absorption cross sections. In the following section we examine the case of NV defects in diamond.

\section{OPTICAL CRYOCOOLING OF NV DEFECTS IN DIAMOND}

We now consider a spherical diamond crystallite of diameter $D$, pumped by a laser beam of cross-sectional area

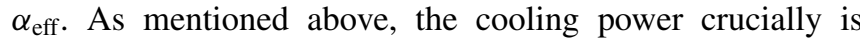
dependent on the absorption and emission cross sections. Precise measurements of these cross sections are difficult due to the uncertainties in estimating the defect number density $N$. However, room-temperature $\mathrm{NV}^{-}$absorption and emission cross sections and coefficients as a function of wavelength have been reported in the literature [16,17]. Using the absorption coefficient measured in [16], and their sample's defect number density $N \sim 2 \times 10^{24} \mathrm{~m}^{-3}$, we obtain an estimate for $\sigma_{\text {abs }}(\lambda)$. However, Han et al. [17] report the emission cross section $\sigma_{\mathrm{se}}(\lambda)$ directly yielding a mean emission wavelength $\lambda_{F} \sim$ $721 \mathrm{~nm}$. Thus, optical refrigeration will require pumping of NV defects with $\lambda>721 \mathrm{~nm}$. The absorption cross section $\sigma_{\text {abs }}(\lambda)$ for $\lambda>\lambda^{*}=670 \mathrm{~nm}$ becomes quite small and, since the cooling efficiency will depend strongly on $\sigma_{\text {abs }}$, we performed experiments to measure this quantity with high precision for $\lambda>\lambda^{*}$ (see the Appendix). These measured cross sections for $\lambda>\lambda^{*}$ were combined with those in the reported literature for $\lambda<\lambda^{*}[16,17]$ to obtain Fig. 1 .

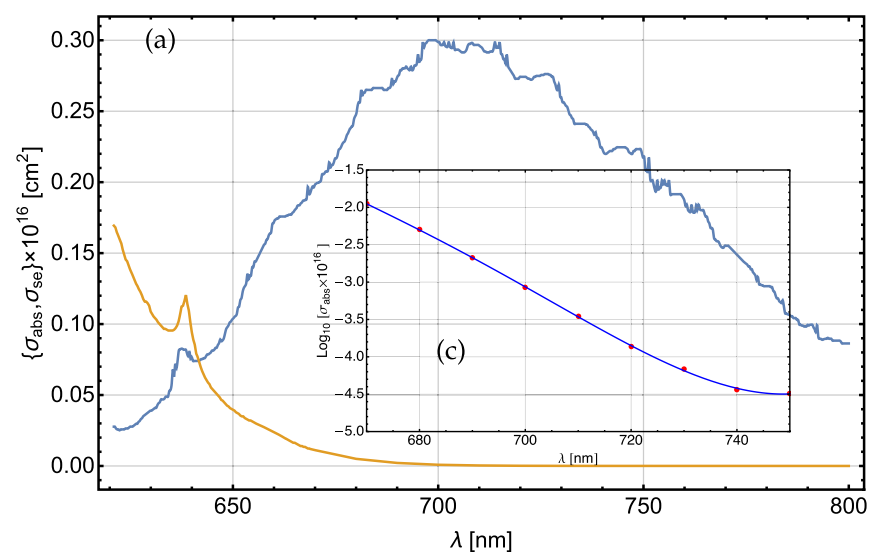

(b)

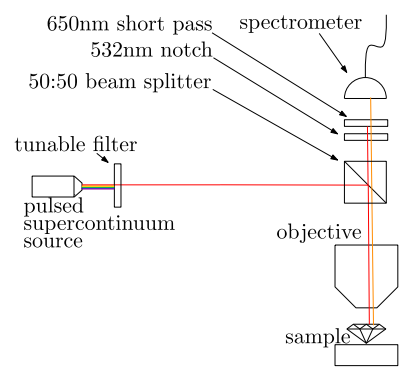

FIG. 1. (a) Room-temperature absorption (yellow) and emission (blue) cross sections for NV diamond over the spectral range of interest for anti-Stokes emission. (b) Schematic of the detailed absorption cross-section measurement setup (see Appendix). (c) Measured absorption cross section displayed on a logarithmic plot (red markers; blue is fitted quartic guide to the eye). 

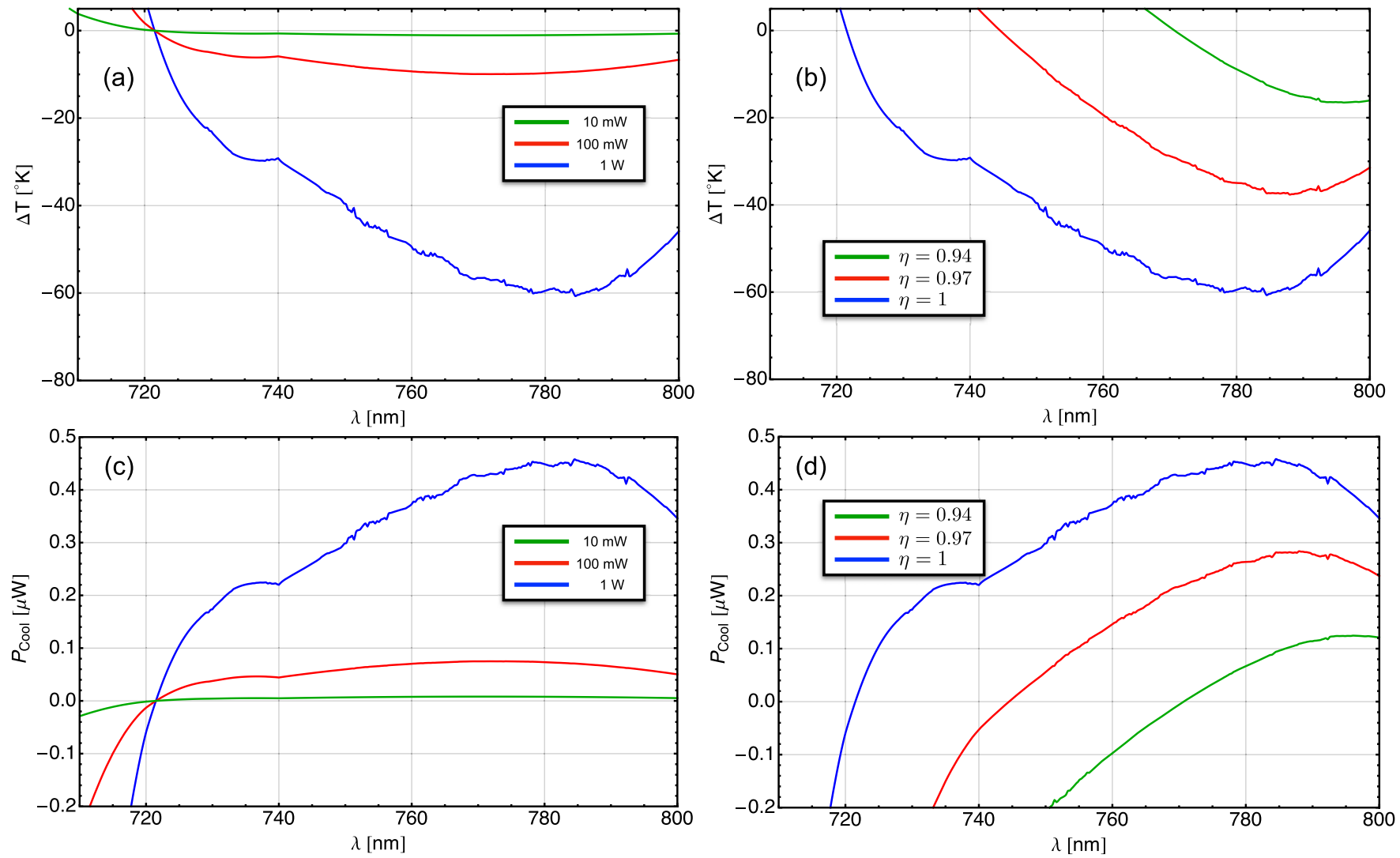

FIG. 2. Cooling of a NV-doped 20- $\mu$ m-diam diamond crystallite suspended in vacuum. (a) We plot the equilibrium decrease in temperature, $\Delta T$, of the diamond from ambient as a function of the cooling laser wavelength $\lambda$, for three choices of the laser power $P$ assuming unit quantum efficiency $\eta=1$. (b) $\Delta T$ for a power of $1 \mathrm{~W}$, as a function of quantum efficiency $\eta$ assuming all the nonradiative decay processes heat the diamond. (c) Cooling power $P_{\text {cool }}$, for same parameters as in (a) assuming unit quantum efficiency. (d) Cooling power as in (b), where we vary the quantum efficiency.

We now consider a spherical diamond of diameter $D$, containing an ensemble of $\mathrm{NV}^{-}$defects with $N \sim 2.65 \times$ $10^{24} \mathrm{~m}^{-3}(\sim 15 \mathrm{ppm})$, pumped by a laser beam of spot radius $r_{s}=5 \mu \mathrm{m}$, wavelength $\lambda$, and power $P$. Taking the optical excited-state decay rate $\gamma_{\mathrm{rad}}^{-1}=12 \mathrm{~ns}$ and unit emissivity $\epsilon_{\text {eff }}=1$, we now must consider the ambient thermal environment of the diamond. This ambient environment will provide a heating (cooling) rate which will attempt to bring the diamond back to the ambient temperature. The largest temperature difference will be obtained when the diamond is the most thermally isolated from its surroundings, i.e., heats or cools due only to blackbody radiation. This situation can only be achieved if the particle is levitated in vacuum and the levitation mechanism (e.g., long-wavelength optical tweezers [18]), does not heat or cool the particle. In the case of vacuum trapping we can use Eq. (3), with a $D=20 \mu \mathrm{m}$ diamond, to obtain the cooling results shown in Fig. 2(a). We note that one can obtain substantial cooling with $100-\mathrm{mW}$ laser irradiation but that levitating nanodiamonds in high vacuum is experimentally challenging (optically trapped NDs in air at atmospheric pressures have been reported [19], whereas using parametric cooling can trap nanodiamonds in high vacuum [20,21]). Researchers initially found that optical levitation of diamonds in air causes extreme heating of the nanodiamonds due, possibly, to burning [22,23]. Recently, however, experiments in nitrogen atmospheres with high-purity diamond showed that nanodiamonds can be optically levitated with no heating or burning [24]. In the above we assumed that the optical cycling of the NV defect contained no nonradiative transitions which could heat the diamond. In fact the quantum efficiency, defined as the ratio of radiative to total decay rates $\eta=\gamma_{\mathrm{rad}} /\left(\gamma_{\mathrm{rad}}+\gamma_{\mathrm{nrad}}\right)$, of $\mathrm{NV}^{-}$in nanodiamonds has been shown to vary considerably between different nanodiamonds (NDs) [25]. We can take heating due to nonradiative processes into account in Eq. (3) through setting $\lambda_{F} \rightarrow \lambda_{F^{*}} \equiv\left[1 / \lambda_{F}-\right.$ $\left.\kappa /\left(h c \gamma_{\mathrm{rad}}\right)\right]^{-1}$, where $\kappa$ quantifies the nonradiative heating and effectively increases the mean emission wavelength $\lambda_{F^{*}} \geqslant \lambda_{F}$ [15]. We now consider the worst-case scenario, where all the nonradiative decay processes heat the diamond, and set $\kappa=$ $\gamma_{\text {nrad }}\left(h c / \lambda_{F}\right)$ to obtain $\lambda_{F^{*}}=\lambda_{F} \times \eta /(2 \eta-1)$. In Fig. 2(b) we graph the equilibrium temperature change for a range of quantum efficiencies and observe that the cooling is very sensitive to the actual quantum efficiency of the NV defects. We note that NV defects with quantum efficiencies ranging between 0.7 and 0.99 have been measured [26-30]. Placing the diamond crystallite on a thermally insulating substrate (Pyrex glass), or optically trapping the diamond in liquid, e.g., water, will reduce this temperature difference between the diamond and its environment. In Figs. 2(c) and 2(d), we plot the corresponding cooling powers obtained for identical situations as in Figs. 2(a) and 2(b). The cooling powers obtained are not as large as those potentially achievable using rare-earth materials; 


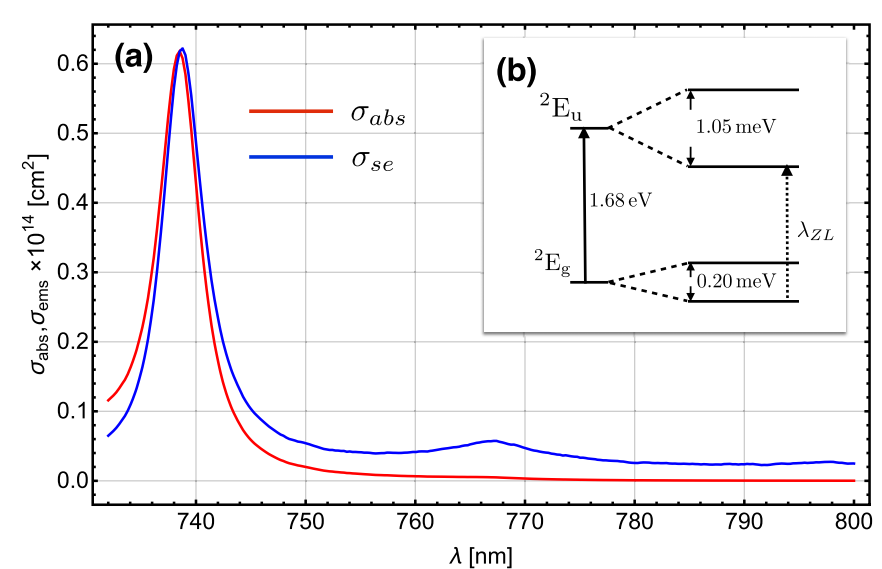

FIG. 3. (a) Room-temperature emission and absorption cross section of the $\mathrm{SiV}^{-}$defect derived from photoluminescence spectra [39] and McCumber theory. (b) Electronic structure of the $\mathrm{SiV}$ defect [35].

however, the latter are typically highly cytotoxic and cannot be used in biological applications [31].

\section{III. $\mathrm{SiV}^{-}$DEFECT CRYOCOOLING}

The negatively charged silicon-vacancy defect, $\mathrm{SiV}^{-}$ [32-37], has attracted much interest recently for potential use in quantum information. Its photophysical and electronic properties have been studied [35,38], and this defect has a particular advantage for cryocooling due to its narrow emission spectrum and very high emission and absorption cross section with an excited-state lifetime of $\tau_{\text {rad }}=1.2 \mathrm{~ns}$ at room temperature. The room-temperature photoluminescence spectra from an individual $\mathrm{SiV}^{-}$defect was reported in [39]. The defect possesses a ground- and excited-state doublet (each of which are doubly degenerate) [35], as shown in Fig. 3(b). We now examine its potential applicability in optical cryocooling. Using Fuchtbauer-Ladenburg theory we can obtain the emission cross section as

$$
\sigma_{\mathrm{se}}(\lambda)=\frac{\lambda^{5} I(\lambda)}{8 \pi n^{2} c \tau_{\mathrm{rad}} \lambda_{F} \Sigma},
$$

where $\lambda_{F} \equiv \frac{1}{\Sigma} \int \lambda I(\lambda) d \lambda$, and $\Sigma \equiv \int I(\lambda) d \lambda$, and the refractive index of diamond is $n=2.4$. From this it is possible to estimate the absorption cross section using reciprocal relations first derived by McCumber [40] (see Eq. (39) in [41] or Eq. (9) in [42], and Sec. 3.2.2 in [43]) as

$$
\sigma_{\mathrm{se}}(\lambda)=\sigma_{\mathrm{abs}}(\lambda) \frac{Z_{l}}{Z_{u}} \exp \left[\frac{\left(E_{Z L}-h c / \lambda\right)}{k_{B} T}\right],
$$

where the partition functions for $Z_{u}$ (upper doublet) and $Z_{l}$ (lower doublet) are given as

$$
Z_{x}=\sum_{k=1}^{2} d_{k}^{x} e^{-E_{k}^{x} / k_{b} T}
$$

where $E_{k}^{x}$ are the energies of the $k^{\prime}$ th level in the $x=u$ upper doublet or $x=l$ lower doublet, with degeneracies $d_{k}^{x}$.

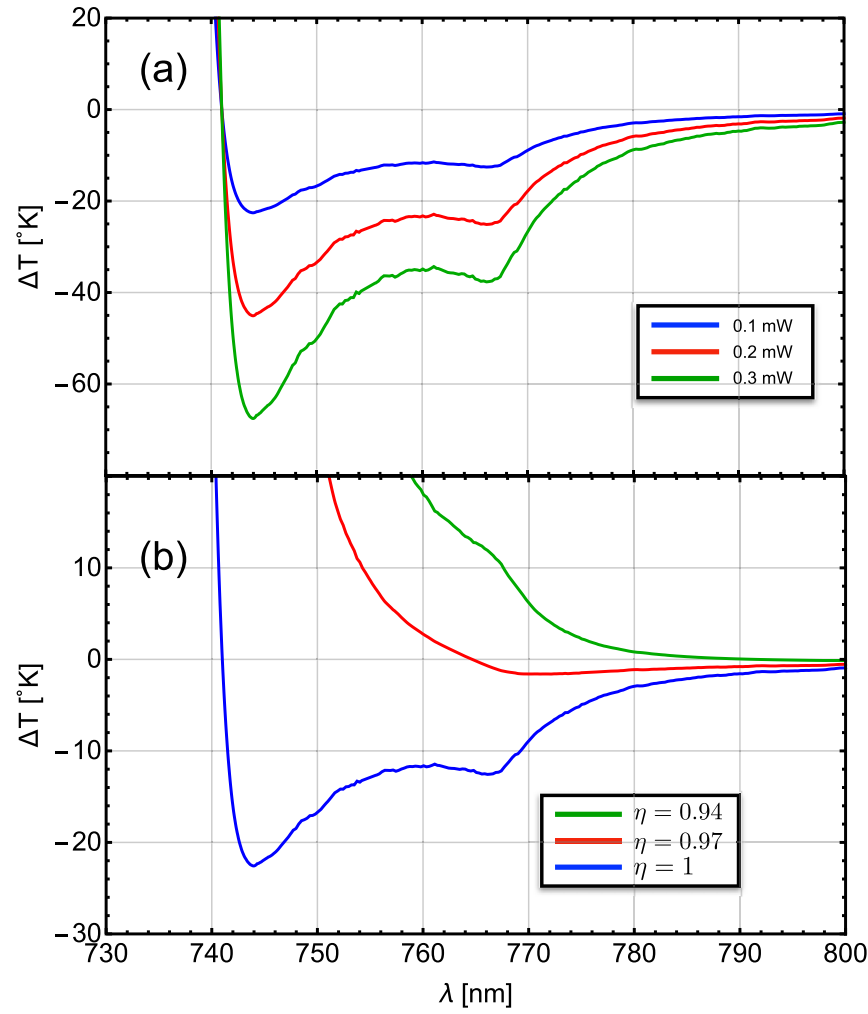

FIG. 4. Cooling of a SiV-doped 20- $\mu$ m-diam microdiamond suspended in vacuum. (a) Equilibrium decrease in temperature, $\Delta T$, of the diamond crystallite from ambient as a function of the cooling laser wavelength $\lambda$, for three choices of the laser power $P$. (b) $\Delta T$ for a power of $0.1 \mathrm{~mW}$, as a function of quantum efficiency $\eta$ assuming all the nonradiative decay processes heat the diamond. We note that experimentally one study reported values for $\eta \sim 0.1$ [38].

Following [43] we obtain

$$
\frac{\sigma_{\mathrm{se}}(\lambda)}{\sigma_{\mathrm{abs}}(\lambda)}=Z_{\text {ratio }} \exp \left[\frac{\epsilon-h c / \lambda}{k_{B} T}\right],
$$

where

$$
Z_{\text {ratio }}^{-1}=\frac{\left(1-e^{-\Delta E_{l} / k_{B} T}\right)}{\left(1-e^{-\Delta E_{u} / k_{B} T}\right)} \frac{\left(1-e^{-d_{u} \Delta E_{u} / k_{B} T}\right)}{\left(1-e^{\left.-d_{l} \Delta E_{l} / k_{B} T\right)}\right.},
$$

where $\Delta E_{l}=0.2 \mathrm{meV}, \Delta E_{u}=1.05 \mathrm{meV}, d_{l}=d_{u}=2$ for the $\mathrm{SiV}$ defect, and $\epsilon=h c / \lambda_{Z L}$. Using Eq. (7) we find the absorption cross section shown in Fig. 3(a). We note that, different from the case of the $\mathrm{NV}^{-}$defect, there is significant spectral overlap between the emission and absorption cross sections and therefore we may expect this defect to exhibit a greater cooling capability.

Using the cross sections shown in Fig. 4, and choosing a SiV defect number density of $N \sim 2.65 \times 10^{23} \mathrm{~m}^{-3}(\sim 1.5 \mathrm{ppm})$, in a relatively large microdiamond of diameter $D=20 \mu \mathrm{m}$ pumped by a laser with spot size $r_{s}=5 \mu \mathrm{m}$, levitated in vacuum with radiative heating from the ambient roomtemperature surroundings we obtain the equilibrium temperature change from Eq. (3), as shown in Fig. 4(a). Here we estimate $\lambda_{F}=741 \mathrm{~nm}$ from Fig. 11 of [33]. From Fig. 4(a) we find that this defect displays tremendous cooling power. The large $\Delta T$ predicted by Eq. (3) assumes that the cross sections 
do not significantly change with temperature. From [39] it is known that the emission cross section of $\mathrm{SiV}$ at $8 \mathrm{~K}$ displays much narrower features and this temperature narrowing in the cross sections is ignored in Eq. (3). However, these changes only occur at low temperatures $(<77 \mathrm{~K})$, and we do not expect much change in the emission and absorption spectra over the 100-300 K temperature ranges which are relevant to this study. In addition we have also assumed no nonradiative heating of the microdiamond, i.e., unit quantum efficiency $\eta=1$. Initial experiments to determine the quantum efficiency of the $\mathrm{SiV}^{-}$ defect in nanodiamonds report quite low values $\eta<0.1$ [38], at room temperature. Bulk values, however, have been reported as large as 0.63 [44]. In Fig. 4(b), we plot $\Delta T$ as a function of $\eta$ assuming $\lambda_{F^{*}}=\lambda_{F} \times \eta /(2 \eta-1)$, i.e., all nonradiative decay heats the microdiamond. To date there have been only one or two experimental studies on the quantum efficiency of the SiV defect either in nanodiamond or bulk form. Much remains to be understood regarding the factors that influence the observed values. However, our study shows that a dramatic reduction in cooling power is associated with even a small decrease in the quantum efficiency from unity. We thus conclude that, unless factors influencing the quantum efficiency of the $\mathrm{SiV}$ defect are better understood, this defect has little possibility to display optical cryocooling.

\section{MEASURING THE TEMPERATURE OF THE DIAMOND CRYSTALLITE}

To verify the optical cryocooling of the diamond crystallite one must be able to accurately measure the temperature of the micron-sized diamond. This is a very challenging task. In the above we have assumed no direct thermal contact between the levitated diamond in vacuum and its surroundings (purely radiative contact), and thus the only methods to measure the temperature of the diamond is either to study the spectrum of the blackbody radiation emitted by the diamond or by analyzing temperature-dependent features $[45,46]$ of the emitted photoluminescence of the defects themselves. The former method is unrealistic as the blackbody powers involved are extremely small while the latter requires continual spectral analysis.

To focus on the latter, particularly in the case of a NV-doped diamond, there is strong dependence on the characteristics of the NV's ZPL and temperature [47-49], and this can be used to determine the particle's temperature with high precision. However, we also now explore an alternative method of sensing the change in temperature which may prove particularly useful in cases when the particle is trapped in liquid, as may be the case in biocompatible applications.

We consider the motional dynamics of the diamond crystallite optically trapped in liquid, the latter held at room temperature, but where the diamond is out of thermal equilibrium, e.g., at an increased or decreased temperature compared to the ambient liquid (see Fig. 5). This temperature difference causes a change in the motional dynamics of the diamond held in the optical trap via a change in the Brownian motion stochastic force. By using the theory of so-called hot or cold Brownian motion [50-56], we predict that the diffusion constant of the diamond will be altered significantly as a function of the diamond's temperature. As the diffusion

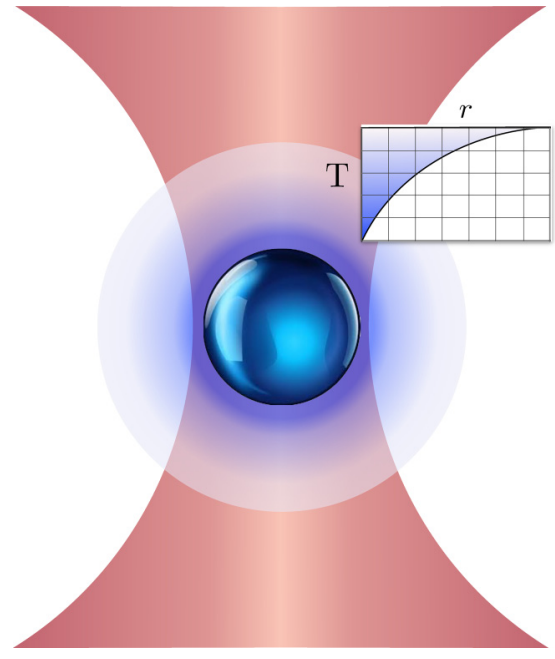

FIG. 5. Schematic illustration of estimating the temperature change of a diamond crystallite undergoing optical cryocooling. We perform optical cryocooling on a diamond (blue sphere) held in an optical tweezer trap (shaded red) in liquid (white). The radial temperature gradient in the liquid (see schematic inset) formed surrounding the diamond creates a situation of cold Brownian motion (CBM), with an associated diffusion constant $D_{\mathrm{CBM}}$. When compared with the Brownian diffusion constant with no cooling, $D_{\mathrm{amb}}$, we find that the ratio $\chi \equiv D_{\mathrm{CBM}} / D_{\mathrm{amb}}$ varies significantly in response to optical cryocooling.

constant can be accurately measured using position and/or velocity, tracking the diamond's temperature can be easily estimated.

We consider the micron-sized diamond crystallite to be held in optical tweezers in the liquid solvent $\mathrm{D}_{2} \mathrm{O}$ (this solvent is chosen for low absorption at relevant optical wavelengths). In a typical optical tweezers setup the fluctuating position or velocity of the diamond is recorded in the signal of a quadrant photodiode. If the diamond is not in thermal equilibrium with the surrounding liquid and is held at a higher or lower temperature, a radial temperature gradient in the liquid is established and the Brownian motion of the particle is modified. This modification is known in the literature as hot or cold Brownian motion [50-56]. In particular, in the case of cold Brownian motion (CBM), the diffusion constant is related to the effective $\mathrm{CBM}$ temperature of the particle, $T_{\mathrm{CBM}}$, and CBM Stokes drag $\gamma_{\mathrm{CBM}}$, via $D_{\mathrm{CBM}}=k_{B} T_{\mathrm{CBM}} / \gamma_{\mathrm{CBM}}[57]$. Here $T_{\mathrm{CBM}}=T_{\mathrm{amb}}+5 \Delta T / 12$, where $\Delta T$ is the change in temperature of the particle from the ambient, and the Stokes drag $\gamma_{\mathrm{CBM}}(T)=6 \pi R \eta_{\mathrm{CBM}}(T)$, where $R$ is the radius of the particle. The temperature-dependent CBM viscosity $\eta_{\mathrm{CBM}}(T)$ is related to the viscosity of the liquid solvent at room temperature, $\eta_{0}$, by

$$
\begin{aligned}
\frac{\eta_{0}}{\eta_{\mathrm{CBM}}(T)} \approx & 1+\frac{193}{486}\left[\ln \left(\frac{\eta_{0}}{\eta_{\infty}}\right)\right]\left[\frac{\Delta T}{\left(T_{\mathrm{amb}}-T_{V F}\right)}\right] \\
& -\left[\frac{56}{243} \ln \left(\frac{\eta_{0}}{\eta_{\infty}}\right)-\frac{12563}{118098} \ln ^{2}\left(\frac{\eta_{0}}{\eta_{\infty}}\right)\right] \\
& \times\left[\frac{\Delta T}{\left(T_{\mathrm{amb}}-T_{V F}\right)}\right]^{2},
\end{aligned}
$$



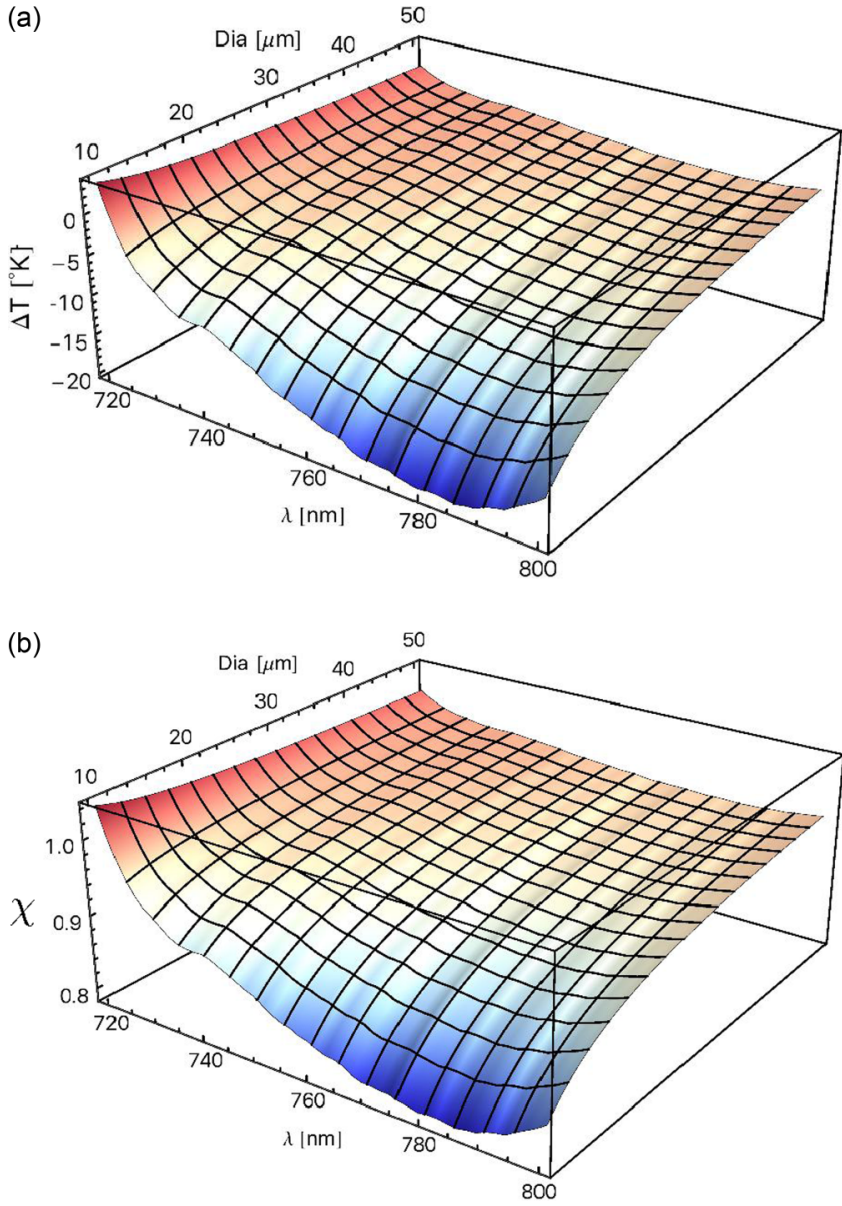

FIG. 6. Temperature change and modification of Brownian motion diffusion constant in a NV-doped diamond crystallite with unit quantum efficiency. (a) NV diamond as in Sec. II: temperature change as a function of illumination wavelength and diamond diameter for a cooling power of $1 \mathrm{~W}$. (b) Dependence of the diffusion constant ratio $\chi=D_{\mathrm{CBM}} / D_{\mathrm{amb}}$.

where for the solvent $\mathrm{D}_{2} \mathrm{O}, \eta_{\infty}=3.456 \times 10^{-5} \mathrm{~Pa} \mathrm{~s}$, $T_{V F}=160 \mathrm{~K}$, and $\eta_{0} \equiv \eta\left(T_{\mathrm{amb}}\right)$, where the temperature dependence of the solvent viscosity is modeled as $\eta(T)=$ $\eta_{\infty} \exp \left[A /\left(T-T_{V F}\right)\right]$, where $A=478.7 \mathrm{~K}$ for $\mathrm{D}_{2} \mathrm{O}$ [57]. To accurately estimate the diffusion constant one must calibrate the quadrant photodiode signal to the actual spatial displacements of the particle, but we instead look at the ratio $\chi=D_{\mathrm{CBM}} / D_{\mathrm{amb}}$, which requires no calibration. We note now that the thermal load is no longer given by Eq. (1), since there is now convective heating of the the cold particle and, instead of Eq. (3), we have

$$
\Delta T=\frac{N \alpha_{\mathrm{eff}} I_{S}}{\pi D\left(4 \epsilon_{\mathrm{eff}} \sigma_{B} T_{\mathrm{amb}}^{3}+h_{\mathrm{cv}}\right)} \frac{\sigma_{\mathrm{abs}}\left(1-\lambda / \lambda_{F}\right)}{1+\sigma_{\mathrm{se}} / \sigma_{\mathrm{abs}}+\alpha_{\mathrm{eff}} I_{S} / P},
$$

where $h_{\mathrm{cv}}$ is the convective heat transfer coefficient for water: $h_{\mathrm{cv}} \sim 30 \mathrm{~W} /\left(\mathrm{m}^{2} \mathrm{~K}\right)$ [58]. In Figs. 6(a) and 6(b) we plot the temperature change $\Delta T$ and diffusion ratio $\chi$ for a range of diamond diameters assuming quantum efficiency $\eta=1$ for the NV-doped diamond (as in Sec. II). From these graphs we observe that the NV-doped diamond exhibits a significant decrease in the ratio $\chi$, particularly for smallerdiameter diamonds, and this should be readily observable in an experiment.

\section{CONCLUSION}

The development of all optical cryocooling has potentially numerous applications ranging from situations which require low power and low vibration, through to refrigeration in difficult environments, e.g., space, high-pressure, and electrical systems, where a liquid coolant is problematic. Traditional materials for optical cryocooling include rare-earth crystals and semiconductors; however, such materials are typically highly toxic in biological environments. In contrast, in this work we have found that diamond doped with nitrogen-vacancy defects shows potential for optical cryocooling. We proposed the optical cryocooling of levitated diamond crystallites which, due to their thermal isolation, may exhibit large temperature reductions using optical cryocooling. For our work we found that cryocooling requires the defects to possess high quantum efficiencies, and NV defects with such high levels of quantum efficiencies have been experimentally demonstrated in nanodiamonds [59], and postselecting nanodiamonds to achieve uniformly high quantum efficiencies is feasible.

Another route to increase the optical quantum efficiency which is particularly suited to the NV defect is to enhance the ZPL emission using the Purcell effect via coupling the defects to an optical cavity. Optical cryocooling is based on the principle that more phonons are absorbed than created. Since the NV center has a strong phonon sideband and hence produces lots of phonons during its radiative decay, cooling effects only start at wavelengths considerably above the ZPL and are highly dependent on quantum efficiency. This could be fundamentally improved by a change of the relative emission pathways associated with the Purcell enhancement of a particular emission wavelength of the NV centers inside a cavity. Purcell enhancement is, by now, an established technique [60-64]. An enhancement of the ZPL has a twofold advantage: The emission on the phonon sideband is reduced relative to the ZPL, which does not create any phonons, and the optical lifetime of the NV is shortened, strengthening the radiative decay relative to any nonradiative pathways, which do not change in their decay rate, i.e., increasing the quantum efficiency. While a NV diamond inside a cavity sets some limitations in terms of applicability it might provide strong advantages for cooling: lowering the effective excitation wavelength above which a cooling effect starts, which in turn increases the absorption cross section, i.e., the cooling effect at the same power as well as improving the cooling effect through an effective increase of the quantum efficiency. This might be an interesting avenue for further improvements.

Thus, we have found that the optical cryocooling of NV-doped diamond crystallites is viable and there may be potential use for this in biocompatible applications, e.g., in cryosurgery or cryotherapy. The use of other defects in diamond with higher Debye-Waller factors and near-unity quantum efficiency would prove even better candidates for optical cryocooling of diamond. 


\section{ACKNOWLEDGMENTS}

The authors thank B. Gibson (RMIT) and K. Xia (MQ) for useful discussions and L. McGuinness (Ulm University), for discussions and provision of a NV-dense diamond sample. This work was supported by the Australian Research Council Centre of Excellence in Engineered Quantum Systems EQUS (Project No. CE110001013) and Australian Research Council Discovery Project No. DP130104381.

\section{APPENDIX}

The absorption cross section of the negatively charged NV center is well known only for the excitation wavelength $\lambda=532 \mathrm{~nm}$. For cryocooling it is important to measure the absorption cross section as a function of wavelength for $\lambda>\lambda^{*}=$ $670 \mathrm{~nm}$ without relying on typically inaccurate estimates for the number of emitting NV centers and the laser spot size. We achieved this by comparing the emission from the $\mathrm{NV}^{-}$centers (zero-phonon-line emission at $637 \mathrm{~nm}$ ) for different excitation wavelengths and relying on the literature value for 532-nm excitation [65]. We ensured that the excitation power was consistently at $200 \mu \mathrm{W}$ for all high wavelengths and accounted for the reduced power of our 532-nm reference measurement. We also ensured that the excitation power is low enough to not reach any saturation within the measurement to guarantee linear behavior with excitation power and comparability across all excitation wavelengths. Different excitation wavelengths were created by a supercontinuum source with a tunable filter (see Fig. 1) and focused onto a single crystal diamond sample with very high NV center density to ensure a good signal. A 650-nm short pass and 532-nm notch blocked the sensor from reflections of the excitation light, which were much stronger than the signal. The incoming light was then directed to a spectrometer and measurements with an acquisition time of 1 $\mathrm{s}$ were taken. We compared the signal at the $\mathrm{NV}^{-}$zero-phonon line, and the relative signals as a function of wavelength were then linearly scaled to an absorption cross section by equating the reference intensity, measured with 532-nm excitation, to the literature value of $0.95 \times 10^{-16} \mathrm{~cm}^{2}$ [65]. Note that this measurement is more precise than an absorption measurement because out of all the possible absorption mechanisms we select only that of the NV centers by measuring the emission of $\mathrm{NV}$ centers rather than the reduction in the transmitted light.
[1] P. Pringsheim, Z. Phys. 57, 739 (1929).

[2] R. I. Epstein, M. I. Buchwald, B. C. Edwards, and T. R. Gosnell, Nature (London) 377, 500 (1995).

[3] D. V. Seletskiy, S. D. Melgaard, S. Bigotta, A. Di Lieto, M. Tonelli, and M. Sheik-Bahae, Nat. Photonics 4, 161 (2010).

[4] J. Zhang, D. Li, R. Chen, and Q. Xiong, Nature (London) 493, 504 (2013).

[5] S. Melgaard, D. Seletskiy, V. Polyak, Y. Asmerom, and M. Sheik-Bahae, Opt. Express 22, 7756 (2014).

[6] M. Sheik-Bahae and R. Epstein, Optical Refrigeration: Science and Applications of Laser Cooling of Solids (Wiley-VCH, Weinheim, 2009).

[7] D. V. Seletskiy, M. P. Hehlen, R. I. Epstein, and M. Sheik-Bahae, Adv. Opt. Photonics 4, 78 (2012).

[8] M. P. Hehlen, M. Sheik-Bahae, R. I. Epstein, S. D. Melgaard, and D. V. Seletskiy, J. Mater. Chem. C 1, 7471 (2013).

[9] G. Nemova and R. Kashyap, J. Phys.: Conf. Ser. 619, 012037 (2015).

[10] Y. Zhu, J. Li, W. Li, Y. Zhang, and X. Yang, Theranostics 2, 302 (2012).

[11] A. Gage and J. Baust, Cryobiology 37, 171 (1998).

[12] D. Theodorescu, Rev. Urol. 6, S9 (2004).

[13] B. Rubinsky, Annu. Rev. Biomed. Eng. 2, 157 (2000).

[14] M. Sheik-Bahae and R. I. Epstein, Laser Photonics Rev. 3, 67 (2009).

[15] X. Luo, M. D. Eisaman, and T. R. Gosnell, Opt. Lett. 23, 639 (1998).

[16] V. M. Acosta, Ph.D. thesis, University of California, Berkeley, 2011 .

[17] K. Y. Han, K. I. Willig, E. Rittweger, F. Jelezko, C. Eggeling, and S. W. Hell, Nano Lett. 9, 3323 (2009).

[18] J. Gieseler, B. Deutsch, R. Quidant, and L. Novotny, Phys. Rev. Lett. 109, 103603 (2012).
[19] L. P. Neukirch, J. Gieseler, R. Quidant, L. Novotny, and A. Nick Vamivakas, Opt. Lett. 38, 2976 (2013).

[20] J. Gieseler, L. Novotny, and R. Quidant, Nat. Phys. 9, 806 (2013).

[21] V. Jain, J. Gieseler, C. Moritz, C. Dellago, R. Quidant, and L. Novotny, Phys. Rev. Lett. 116, 243601 (2016).

[22] L. P. Neukirch, E. von Haartman, J. M. Rosenholm, and A. N. Vamivakas, Nat. Photonics 9, 653 (2015).

[23] T. M. Hoang, J. Ahn, J. Bang, and T. Li, Nat. Commun. 7, 12250 (2016).

[24] A. C. Frangeskou, A. T. M. A. Rahman, L. Gines, S. Mandal, O. A. Williams, P. F. Barker, and G. W. Morley, arXiv:1608.04724.

[25] M. Frimmer, A. Mohtashami, and A. Femius Koenderink, Appl. Phys. Lett. 102, 121105 (2013).

[26] A. Gruber, A. Drabenstedt, C. Tietz, L. Fleury, J. Wrachtrup, and C. von Borczyskowski, Science 276, 2012 (1997).

[27] E. Rittweger, K. Y. Han, S. E. Irvine, C. Eggeling, and S. W. Hell, Nat. Photonics 3, 144 (2009).

[28] G. Waldherr, J. Beck, M. Steiner, P. Neumann, A. Gali, T. H. Frauenheim, F. Jelezko, and J. Wrachtrup, Phys. Rev. Lett. 106, 157601 (2011).

[29] S. Schietinger, M. Barth, T. Alchele, and O. Benson, Nano Lett. 9, 1694 (2009).

[30] F. A. Inam, M. D. W. Grogan, M. Rollings, T. Gaebel, J. M. Say, C. Bradac, T. A. Birks, W. J. Wadsworth, S. Castelletto, J. R. Rabeau, and M. J. Steel, ACS Nano 7, 3833 (2013).

[31] K. T. Rim, K. H. Koo, and J. S. Park, Saf. Health Work 4, 12 (2013).

[32] J. P. Goss, R. Jones, S. J. Breuer, P. R. Briddon, and S. Öberg, Phys. Rev. Lett. 77, 3041 (1996).

[33] K. Iakoubovskii and G. J. Adriaenssens, Phys. Rev. B 61, 10174 (2000). 
[34] C. Hepp, T. Müller, V. Waselowski, J. N. Becker, B. Pingault, H. Sternschulte, D. Steinmüller-Nethl, A. Gali, J. R. Maze, M. Atatüre, and C. Becher, Phys. Rev. Lett. 112, 036405 (2014).

[35] L. J. Rogers, K. D. Jahnke, M. W. Doherty, A. Dietrich, L. P. McGuinness, C. Müller, T. Teraji, H. Sumiya, J. Isoya, N. B. Manson, and F. Jelezko, Phys. Rev. B 89, 235101 (2014).

[36] A. Sipahigil, K. D. Jahnke, L. J. Rogers, T. Teraji, J. Isoya, A. S. Zibrov, F. Jelezko, and M. D. Lukin, Phys. Rev. Lett. 113, 113602 (2014).

[37] L. J. Rogers, K. D. Jahnke, M. H. Metsch, A. Sipahigil, J. M. Binder, T. Teraji, H. Sumiya, J. Isoya, M. D. Lukin, P. Hemmer, and F. Jelezko, Phys. Rev. Lett. 113, 263602 (2014).

[38] E. Neu, M. Agio, and C. Becher, Opt. Express 20, 19956 (2012).

[39] A. Dietrich, K. D. Jahnke, J. M. Binder, T. Teraji, J. Isoya, L. J. Rogers, and F. Jelezko, New J. Phys. 16, 113019 (2014).

[40] D. E. McCumber, Phys. Rev. 136, A954 (1964).

[41] C. E. Mungan and T. R. Gosnell, in Advances in Atomic, Molecular, and Optical Physics, edited by B. Benjamin and W. Herbert (Academic, San Diego, CA, 1999), p. 161.

[42] K. Ouannes, M. T. Soltani, M. Poulain, and G. Boulon, J. Alloys Compd. 603, 132 (2014).

[43] A. S. Vemuru, Master's thesis, Russ College of Engineering and Technology, Ohio University, 2008.

[44] J. Riedrich-Möller, C. Arend, C. Pauly, F. Mücklich, M. Fischer, S. Gsell, M. Schreck, and C. Becher, Nano Lett. 14, 5281 (2014).

[45] G. Kucsko, P. C. Maurer, N. Y. Yao, M. Kubo, H. J. Noh, P. K. Lo, H. Park, and M. D. Lukin, Nature (London) 500, 54 (2013).

[46] P. Neumann, I. Jakobi, F. Dolde, C. Burk, R. Reuter, G. Waldherr, J. Honert, T. Wolf, A. Brunner, J. H. Shim, D. Suter, H. Sumiya, J. Isoya, and J. Wrachtrup, Nano Lett. 13, 2738 (2013).

[47] T. Plakhotnik, M. W. Doherty, J. H. Cole, R. Chapman, and N. B. Manson, Nano Lett. 14, 4989 (2014).

[48] T. Plakhotnik, H. Aman, and H.-C. Chang, Nanotechnology 26, 245501 (2015).

[49] W. W.-W. Hsiao, Y. Y. Hui, P.-C. Tsai, and H.-C. Chang, Acc. Chem. Res. 49, 400 (2016).
[50] D. Rings, R. Schachoff, M. Selmke, F. Cichos, and K. Kroy, Phys. Rev. Lett. 105, 090604 (2010).

[51] D. Rings, M. Selmke, F. Cichos, and K. Kroy, Soft Matter 7, 3441 (2011).

[52] D. Chakraborty, M. V. Gnann, D. Rings, J. Glaser, F. Otto, F. Cichos, and K. Kroy, Europhys. Lett. 96, 60009 (2011).

[53] J. Millen, T. Deesuwan, P. Barker, and J. Anders, Nat. Nanotechnol. 9, 425 (2014).

[54] G. Falasco, M. V. Gnann, D. Rings, and K. Kroy, Phys. Rev. E 90, 032131 (2014).

[55] P. B. Roder, B. Smith, X. Zhou, M. J. Crane, and P. J. Pauzauskie, in Proceedings of SPIE 9380, Laser Refrigeration of Solids VIII, edited by R. I. Epstein, D. V. Seletskiy, and M. Sheik-Bahae (International Society for Optics and Photonics, San Francisco, US, 2015), p. 938007.

[56] P. Roder, B. Smith, and P. Pauzauskie, in Optical Trapping Applications, in the Proceedings of Optics in the Life Sciences (OSA, Vancouver, Canada, 2015).

[57] P. B. Roder, B. E. Smith, X. Zhou, and M. J. Crane, arXiv: 1503.07265 .

[58] S. Kakac, Y. Yener, and A. Pramuanjaroenkij, Sadik Kakac, Yaman Yener, Anchasa (CRC Press, Boca Raton, FL, 2013).

[59] A. Mohtashami and A. F. Koenderink, New J. Phys. 15, 043017 (2013).

[60] C.-H. Su, A. D. Greentree, and L. C. L. Hollenberg, Opt. Express 16, 6240 (2008).

[61] C.-H. Su, A. D. Greentree, and L. C. L. Hollenberg, Phys. Rev. A 80, 052308 (2009).

[62] H. Kaupp, C. Deutsch, H.-C. Chang, J. Reichel, T. W. Hänsch, and D. Hunger, Phys. Rev. A 88, 053812 (2013).

[63] J. Riedrich-Möller, S. Pezzagna, J. Meijer, C. Pauly, F. Mücklich, M. Markham, A. M. Edmonds, and C. Becher, Appl. Phys. Lett. 106 (2015).

[64] S. A. Wolf, I. Rosenberg, R. Rapaport, and N. Bar-Gill, Phys. Rev. B 92, 235410 (2015).

[65] R. Chapman and T. Plakhotnik, Chem. Phys. Lett. 507, 190 (2011). 